\title{
Avaliação da Qualidade de Vida de Pacientes Oncológicos em Cuidados Paliativos
}

doi: https://doi.org/10.32635/2176-9745.RBC.2020v66n3.1122

\author{
Evaluation of the Quality of Life of Oncological Patients in Palliative Care \\ Evaluación de la Calidad de Vida de los Pacientes Oncológicos en Atención Paliativa
}

\author{
Islany Barbosa Soares da Silva'; José de Ribamar Medeiros Lima Júnior²; Joelson dos Santos Almeida³; Dayara Sthéfane Pereira \\ Cutrim4; Ana Hélia de Lima Sardinha ${ }^{5}$
}

\begin{abstract}
RESUMO
Introduçáo: Os cuidados paliativos para pacientes oncológicos têm por finalidade promover a qualidade de vida por meio do controle de sinais e sintomas, melhoria do bem-estar físico, emocional, social e espiritual. Objetivo: Avaliar a qualidade de vida de pacientes oncológicos em cuidados paliativos. Método: Trata-se de uma pesquisa descritiva, transversal, de abordagem quantitativa, realizada com 21 pacientes internados em uma unidade de cuidados paliativos. Foram coletados dados sobre aspectos sociodemográficos e clínicos, e utilizada a escala do European Organization for Research and Treatment of Cancer Quality-of-Life Questionnaire Core15 PAL (EORTC QLQ C-15 PAL) para avaliação da qualidade de vida. Resultados: A média de saúde global foi de 60,32, sendo a qualidade de vida considerada satisfatória. $\mathrm{Na}$ escala de sintomas, os domínios mais afetados foram dor $(52,38)$, constipação $(46,03)$ e fadiga $(42,86)$. A função emocional $(37,30)$ mostrou-se pior do que a avaliação da função física $(59,79)$. Houve relação significativa do tempo de diagnóstico com a qualidade de vida geral e a dispneia do performance status de Karnofsky (KPS) com o funcionamento físico, bem como da presença de metástase com dispneia. Conclusáo: A qualidade de vida deve ser avaliada diariamente, visto que os cuidados paliativos consideram, além dos sintomas, o conforto do próprio paciente durante o estado de doença que ameaça à vida e as intervençóes efetivas de cuidados, permitindo assim o direcionamento da atuação da equipe multiprofissional.
\end{abstract}

Palavras-chave: Cuidados Paliativos; Qualidade de Vida; Avaliação de Estado de Karnofsky; Neoplasias.

\section{ABSTRACT}

Introduction: Palliative care for cancer patients aims to promote quality of life, by controlling signs and symptoms, improving physical, emotional, social and spiritual well-being. Objective: To evaluate the quality of life of cancer patients in palliative care. Method: Descriptive, cross-sectional, quantitative approach study, conducted with 21 patients admitted to a palliative care unit. Data on sociodemographic and clinical aspects were collected, using the European Organization for Research and Treatment of Cancer Quality-of-Life Questionnaire Core15 PAL (EORTC QLQ C-15) PAL scale to assess quality of life. Results: The average global health was 60.32 , with quality of life considered satisfactory. In the symptom scale, the most affected domains were pain (52.38), constipation (46.03) and fatigue (42.86). Emotional function (37.30) was assessed worse than physical function (59.79). There was a significant relationship between the time of diagnosis with the general quality of life and dyspnea, of Karnofsky (KPS) performance status, with physical functioning, and presence of metastasis with dyspnea. Conclusion: Quality of life should be assessed daily, as palliative care considers, in addition to symptoms, the patient's own comfort during a life-threatening illness and effective care interventions, helping the multiprofessional team to focus their action.

Key words: Palliative Care; Quality of Life; Karnofsky Performance Status; Neoplasms.

\section{RESUMEN}

Introducción: Los cuidados paliativos para pacientes con cáncer tienen como objetivo promover la calidad de vida, controlando los signos y síntomas, mejorando el bienestar físico, emocional, social y espiritual. Objetivo: evaluar la calidad de vida de los pacientes con cáncer en cuidados paliativos. Método: Este es un enfoque descriptivo, transversal y cuantitativo, realizado con 21 pacientes ingresados en una unidad de cuidados paliativos. Se recopilaron datos sobre aspectos sociodemográficos y clínicos, utilizando la escala del European Organization for Research and Treatment of Cancer Quality-of-Life Questionnaire Core15 PAL (EORTC QLQ C-15 PAL) para evaluar la calidad de vida. Resultados: La salud global promedio fue de 60.32 , con calidad de vida considerada satisfactoria, en la escala de síntomas los dominios más afectados fueron dolor (52.38), estreńimiento (46.03) y fatiga (42.86). La función emocional (37.30) se evaluó peor que la función física (59.79). Hubo una relación significativa entre el momento del diagnóstico y la calidad de vida general y la disnea, del performance status de Karnofsky (KPS) con el funcionamiento físico, así como la presencia de metástasis con disnea. Conclusión: La calidad de vida debe evaluarse diariamente, como lo consideran los cuidados paliativos, además de los síntomas, así como la propia comodidad del paciente durante una enfermedad potencialmente mortal e intervenciones de atención efectivas, lo que permite la dirección del desempeño del equipo multiprofesional.

Palabras clave: Cuidados Paliativos; Calidad de Vida; Estado de Ejecución de Karnofsky; Neoplasias.

\footnotetext{
${ }^{1}$ Enfermeira. Residente em Oncologia. Residência Multiprofissional em Oncologia da Secretaria de Saúde do Estado do Maranhão (SES-MA). São Luís (MA), Brasil. Orcid iD: https://orcid.org/0000-0002-1134-9553

${ }^{2}$ Enfermeiro. Doutorando em Ciências da Saúde pela Universidade Federal do Maranhão (UFMA). Professor-Assistente A da UFMA no Curso de Enfermagem do Campus Pinheiro. São Luís (MA), Brasil. Orcid iD: https://orcid.org/0000-0001-9172-3682

${ }^{3}$ Enfermeiro. Mestrando em Saúde e Ambiente pela UFMA. Docente do Programa de Residência Multiprofissional em Oncologia da SES-MA. São Luís (MA), Brasil. Orcid iD: https://orcid.org/0000-0001-6926-7043

${ }^{4}$ Enfermeira. Residente em Oncologia. Residência Multiprofissional em Oncologia da SES-MA. São Luís (MA), Brasil. Orcid iD: https://orcid.org/0000-0002-1134-9553 ${ }^{5}$ Enfermeira. Doutora em Ciências Pedagógicas. Professora-Titular do Departamento de Enfermagem da UFMA. São Luís (MA), Brasil. Orcid iD: https://orcid. org/0000-0002-8720-6348

Endereço para correspondência: Islany Barbosa Soares da Silva. Rua Duque Bacelar, S/No - Parque dos Nobres. São Luís (MA), Brasil. CEP 65074-253. E-mail: islanybarbosa@gmail.com
} 


\section{INTRODUÇÃO}

As doenças crônicas não transmissíveis (DCNT) são as maiores causas de morbimortalidade no mundo, ocasionando cerca de $63 \%$ das mortes ${ }^{1}$. Entre as DCNT, estão as doenças cardiovasculares, doenças respiratórias, diabetes e o câncer. Essas doenças refletem diretamente na perda da qualidade de vida, sobretudo o câncer, ao deixar as pessoas com limitaçóes e incapacidades ${ }^{2,3}$.

Nesse contexto, apesar dos avanços tecnológicos no setor da saúde, com melhores recursos diagnósticos e terapêuticos e a ampliaçáo do rastreamento, em muitos casos, o diagnóstico do câncer ainda é tardio. Desse modo, geralmente a cura está relacionada ao estádio da doença; assim, quanto mais precoce for o diagnóstico, maiores serão as chances de cura ${ }^{4,5}$.

No momento da realização do diagnóstico, é fundamental identificar o estadiamento do câncer, o que possibilita a definição mais adequada do tratamento e a avaliação do prognóstico. Assim, para os pacientes que se encontram em estádios III ou IV, o câncer é definido como avançado, a indicação do tratamento passa a ser paliativo, visando, sobretudo, ao controle de sintomas e ao aumento da sobrevida; como a realização de quimioterapia e radioterapia paliativas, sendo as possibilidades de cura com o tratamento reduzidas ${ }^{5-7}$.

Apesar de a melhor efetividade do tratamento colaborar com o aumento da sobrevida e o controle da doença, principalmente por meio da inclusão de novas terapêuticas, como a imunoterapia e hormonioterapia, tanto a própria doença quanto o tratamento geram muito sofrimento para os pacientes e seus familiares, sobretudo nos casos avançados ${ }^{8}$.

Assim, para os pacientes que se encontram fora de possibilidades terapêuticas curativas, o tratamento precisa estar voltado para o controle de sinais e sintomas pouco controláveis, como dor, náuseas, vômitos, anorexia, fadiga, depressão, ansiedade, constipação, entre outros? Nessa perspectiva, surge o cuidado paliativo como uma abordagem que visa a dar suporte aos pacientes e familiares, promovendo o aumento da qualidade de vida, por meio do controle de sintomas físicos, psicológicos, sociais e espirituais ${ }^{10,11}$.

A abordagem dos cuidados paliativos é indicada desde o diagnóstico para todos os pacientes com doenças graves, progressivas e incuráveis, as quais ameacem à continuidade da vida. Portanto, os pacientes com câncer devem ter os cuidados paliativos integrados ao tratamento antineoplásico desde o diagnóstico e não apenas no final da vida. A terapêutica paliativa busca promover uma sobrevivência pelo maior tempo possível com preservação da qualidade de vida ${ }^{12}$.
A Organização Mundial da Saúde (OMS) define cuidados paliativos como:

uma abordagem que melhora a qualidade de vida de pacientes (adultos e crianças) e seus familiares, que enfrentam doenças que ameacem à vida. Previne e alivia o sofrimento por intermédio da identificaçáo precoce, avaliaçáo correta e tratamento da dor e outros problemas físicos, psíquicos, sociais, familiares e espirituais ${ }^{13}$.

A qualidade de vida é um conceito amplo e subjetivo. Diversos fatores devem ser determinados para avaliar a qualidade de vida, os quais excedem aspectos referentes à doença e ao tratamento, abrangendo as dimensôes física, funcional, emocional, familiar, social e espiritual ${ }^{9,14}$. A OMS define qualidade de vida como "a percepçáo do indivíduo de sua posição na vida, no contexto da cultura e sistemas de valores nos quais ele vive em relação aos seus objetivos, expectativas, padróes e preocupaçóes"15.

Levando em consideração a importância dos cuidados paliativos para a melhoria da qualidade de vida de pacientes com câncer em estágio avançado sem possibilidades de tratamento modificador da doença e para o controle de sintomas que interferem nessa qualidade de vida, esta pesquisa teve como objetivo avaliar a qualidade de vida de pacientes oncológicos em cuidados paliativos.

\section{MÉTODO}

Trata-se de uma pesquisa descritiva, do tipo transversal, com uma abordagem quantitativa, de amostragem não probabilística, por conveniência. A pesquisa foi realizada na unidade de cuidados paliativos de um hospital de referência estadual no tratamento de câncer na cidade de São Luís, Estado do Maranhão, que presta atendimento pelo Sistema Único de Saúde (SUS). Foram incluídos pacientes diagnosticados com câncer sem possibilidades de tratamento modificador da doença, que se encontravam internados na unidade e que estavam em cuidados paliativos exclusivos, apresentavam-se conscientes, lúcidos e com capacidade de verbalizar, com idade igual ou superior a 18 anos e que concordaram em participar da pesquisa. Foi utilizado como critério de exclusão pacientes que não tinham condiçôes clínicas para o preenchimento do instrumento. Assim, participaram da pesquisa 21 pacientes.

O período de coleta de dados se deu entre os meses de junho e dezembro de 2019. Para a coleta de dados, após a confirmação dos critérios de inclusão e exclusão, os pacientes foram abordados individualmente no próprio quarto em que estavam internados, em razão das limitaçôes físicas que dificultavam o seu deslocamento. $\mathrm{O}$ 
Termo de Consentimento Livre e Esclarecido (TCLE) foi entregue aos indivíduos para leitura e esclarecimento de possíveis dúvidas. Após a concordância em participar da pesquisa, os pacientes assinaram e autorizaram o uso das informaçóes de prontuários e posteriormente a aplicação dos instrumentos. A coleta de dados aconteceu em dois momentos. No primeiro momento, aplicou-se um formulário por meio de entrevista; quando necessário, os prontuários eram verificados para levantamento de dados sobre aspectos sociodemográficos e clínicos; o segundo momento consistiu na aplicação do questionário European Organization for Research and Treatment of Cancer Qualityof-Life Questionnaire Core15 PAL (EORTC QLQ C-15 $\mathrm{PAL}$ ) para mensurar a qualidade de vida. Vale destacar que foi solicitada e concedida a autorizaçâo por parte do grupo EORTC para a utilizaçáo do instrumento nesta pesquisa.

As variáveis sociodemográficas e clínicas coletadas foram: idade, sexo, cor, estado civil, escolaridade, profissão, religião, renda familiar, município de residência, diagnóstico, presença ou ausência de metástase, estadiamento clínico, performance status de Karnofsky (KPS), tipo de tratamento realizado, tempo de diagnóstico e tempo de inclusão em cuidados paliativos exclusivos.

A escala de Karnofsky é composta por 11 níveis de "performance", que vão de 0 a 100 , divididos em intervalos de 10, sendo que o " 0 " indica morte e o " 100 " a performance normal, sem alteraçôes por causa da doença ${ }^{16}$.

O questionário EORTC QLQ-C15-PAL é um instrumento composto por 15 itens relacionados à qualidade de vida, os itens de 1 a 3 envolvem o funcionamento físico, com perguntas sobre dificuldades para caminhar, se permanece o dia todo na cama ou cadeira e atividades de vida diária; os itens de 4 a 12 referem-se a alguns sintomas físicos, como dispneia, dor, dificuldades para dormir, fraqueza, falta de apetite, náuseas, constipação, cansaço; os itens 13 e 14 se referem ao estado emocional, com questôes sobre nervosismo e depressão; e o último item é sobre qualidade de vida em geral. Cada item recebe pontuaçáo de $1 \mathrm{a} 4 \mathrm{em}$ uma escala do tipo Likert $(1=$ náo, $2=$ pouco, $3=$ moderadamente, 4 = muito), com exceção do último, em que a pontuação varia de 1 (péssima) a 7 (ótima). A pontuação varia de 0 a 100, sendo que, nas escalas de funcionamento físico e emocional, uma pontuação mais elevada significa uma melhor qualidade de vida, assim como no item 15 , sobre qualidade de vida geral; em contrapartida, nas escalas de sintomas, nos itens de 4 a 12, uma pontuaçáo mais elevada constitui um pior comprometimento da função e maior presença de sintomas ${ }^{17,18}$.

A análise dos dados foi realizada por meio do programa Stata, versão 14.0. Inicialmente, foi feita análise descritiva dos dados, com a distribuição das frequências, médias e desvios-padrão para as variáveis numéricas; e as proporçôes, para as variáveis categóricas. Os escores do questionário EORTC QLQ-C15-PAL foram calculados de acordo com as fórmulas desenvolvidas pelo grupo que criou o questionário, e expressos por meio de médias e desvios-padrão. Posteriormente, realizou-se a comparação das médias dos escores obtidos na EORTC QLQ-C15-PAL com as variáveis sociodemográficas e clínicas. As que apresentavam distribuição normal foram verificadas por meio do teste de Shapiro Wilk. Utilizou-se o teste $\mathrm{T}$ de Student para as variáveis com duas categorias ou a análise de variância para as que apresentavam mais de duas categorias. No caso de não apresentarem distribuição normal, os testes utilizados foram Mann-Whitney para as variáveis com duas categorias ou Kruskall-Wallis nos casos de mais de duas categorias. Para a análise, considerou-se o nível de significância de $5 \%(\mathrm{p} \leq 0,05)$, com confiabilidade de $95 \%$.

Esta pesquisa foi apreciada pelo Comitê de Ética em Pesquisa (CEP) do Hospital Carlos Macieira, obtendo aprovação sob o número CAAE: 05337218.3.0000.8907 e parecer: 3.216.493, sendo realizada dentro das normas éticas da Resolução número 466/2012 e número 510/2016 do Conselho Nacional de Saúde (CNS).

\section{RESULTADOS}

Fizeram parte desta pesquisa 21 pacientes que atenderam aos critérios de inclusão e manifestaram interesse em participar. Destes, houve predominância do sexo feminino $(71,4 \%)$, com faixa etária de 30 a 59 anos $(57,1 \%)$, de cor parda $(85,7 \%)$, com ensino fundamental $(57,1 \%)$ e renda de um a dois salários $(71,4 \%)$. Com relação à procedência, a maioria dos pacientes $(52,4 \%)$ era do interior do Maranhão, e a ocupação $(33,3 \%)$ foi lavradores (Tabela 1).

Conforme a Tabela 2, os cânceres mais prevalentes na populaçáo estudada foram os ginecológicos $(23,8 \%)$, do sistema gastrointestinal $(19,1 \%)$ e mama $(14,3 \%)$; grande parte dos pacientes apresentava metástases a distância $(57,1 \%)$; a maioria tinha um tempo de diagnóstico maior do que 12 meses $(52,4 \%)$ e um tempo de admissão em cuidados paliativos menor do que seis meses $(95,2 \%)$; e 47,6\% dos pacientes apresentaram um KPS de 50\%.

Pode-se perceber que um número expressivo de pacientes náo chegou a realizar nenhum tratamento $(33,3 \%)$. Dos que realizaram algum tratamento, prevaleceram quimioterapia e radioterapia $(19,1 \%)$ e quimioterapia, radioterapia e cirurgia $(19,1 \%)$, conforme a Tabela 3.

Com relação aos escores do questionário EORTC QLQ-C15-PAL, a média de saúde global foi de 60,32. 
Tabela 1. Distribuição das variáveis sociodemográficas dos pacientes com câncer em cuidados paliativos. São Luís-MA, Brasil, 2019

\begin{tabular}{|c|c|c|c|}
\hline Variável & Categoria & $\mathbf{N}$ & $\%$ \\
\hline \multirow{2}{*}{ Sexo } & Feminino & 15 & 71,4 \\
\hline & Masculino & 6 & 28,6 \\
\hline \multirow{3}{*}{ Idade (anos) } & 18 a 29 anos & 1 & 4,8 \\
\hline & 30 a 59 anos & 12 & 57,1 \\
\hline & $\geq 60$ anos & 8 & 38,1 \\
\hline \multirow{2}{*}{ Cor } & Parda & 18 & 85,7 \\
\hline & Negra & 3 & 14,3 \\
\hline \multirow{3}{*}{ Estado civil } & Solteiro & 9 & 42,9 \\
\hline & Casado & 9 & 42,9 \\
\hline & Viúvo & 3 & 14,2 \\
\hline \multirow{4}{*}{ Escolaridade } & Não escolarizado & 2 & 9,5 \\
\hline & Ensino fundamental & 12 & 57,1 \\
\hline & Ensino médio & 6 & 28,6 \\
\hline & Ensino superior & 1 & 4,8 \\
\hline \multirow{2}{*}{ Religião } & Católica & 8 & 38,1 \\
\hline & Evangélica & 13 & 61,9 \\
\hline \multirow{3}{*}{ Renda } & <1 salário mínimo & 4 & 19,1 \\
\hline & 1 a 2 salários mínimos & 15 & 71,4 \\
\hline & 2 a 4 salários mínimos & 2 & 9,5 \\
\hline \multirow{3}{*}{ Procedência } & São Luís & 9 & 42,9 \\
\hline & Interior do Estado & 11 & 52,4 \\
\hline & Outros Estados & 1 & 4,7 \\
\hline \multirow{5}{*}{$\begin{array}{l}\text { Profissão/ } \\
\text { ocupação }\end{array}$} & Aposentado & 4 & 19,1 \\
\hline & Do lar & 3 & 14,3 \\
\hline & Lavrador & 7 & 33,3 \\
\hline & Professor & 2 & 9,5 \\
\hline & Outras & 5 & 23,8 \\
\hline
\end{tabular}

Tabela 2. Distribuição das variáveis dos aspectos clínicos de pacientes com câncer em cuidados paliativos. São Luís-MA, Brasil, 2019

\begin{tabular}{l|l|c|c}
\hline Variável & Categoria & $\mathbf{N}$ & $\%$ \\
\hline \multirow{4}{*}{ Diagnóstico } & Ginecológicos & 5 & 23,8 \\
\cline { 2 - 4 } & Gastrointestinais & 4 & 19,1 \\
\cline { 2 - 4 } & Mama & 3 & 14,3 \\
\cline { 2 - 4 } & Outros & 9 & 42,9 \\
\hline \multirow{3}{*}{ Metástase a distância } & Sim & 12 & 57,1 \\
\cline { 2 - 4 } & Não & 9 & 42,9 \\
\hline \multirow{4}{*}{ Tempo de diagnóstico } & $<6$ meses & 8 & 38,1 \\
\cline { 2 - 4 } & De 6 a 12 meses & 2 & 9,5 \\
\cline { 2 - 4 } & $>12$ meses & 11 & 52,4 \\
\hline \multirow{2}{*}{$\begin{array}{l}\text { Tempo de admissão } \\
\text { em cuidados paliativos }\end{array}$} & $<6$ meses & 20 & 95,2 \\
\cline { 2 - 4 } & De 6 a 12 meses & 1 & 4,8 \\
\hline \multirow{4}{*}{$\begin{array}{l}\text { Performance status de } \\
\text { Karnofsky }\end{array}$} & $30 \%$ & 3 & 14,3 \\
\cline { 2 - 4 } & $40 \%$ & 6 & 28,6 \\
\cline { 2 - 4 } & $50 \%$ & 10 & 47,6 \\
\cline { 2 - 4 } & $60 \%$ & 2 & 9,5 \\
\hline
\end{tabular}

Tabela 3. Distribuição do tipo de tratamento realizado pelos pacientes com câncer em cuidados paliativos. São Luís-MA, Brasil, 2019

\begin{tabular}{l|c|c}
\hline Tratamento & N & $\%$ \\
\hline Nenhum tratamento & 7 & 33,3 \\
\hline Quimioterapia & 2 & 9,5 \\
\hline Cirurgia & 1 & 4,8 \\
\hline Quimioterapia e radioterapia & 4 & 19,1 \\
\hline Quimioterapia; radioterapia e cirurgia & 4 & 19,1 \\
\hline $\begin{array}{l}\text { Quimioterapia; hormonioterapia e } \\
\text { cirurgia }\end{array}$ & 1 & 4,8 \\
\hline Radioterapia e cirurgia & 1 & 4,8 \\
\hline $\begin{array}{l}\text { Quimioterapia; hormonioterapia; } \\
\text { cirurgia e radioterapia }\end{array}$ & 1 & 4,8 \\
\hline
\end{tabular}

Pode-se perceber ainda que, entre as escalas de sintomas, o domínio mais afetado foi a dor $(52,38)$, seguido de constipaçấo $(46,03)$ e fadiga $(42,86)$. Nas escalas funcionais, o comprometimento do funcionamento emocional $(37,30)$ obteve uma média mais baixa do que o funcionamento físico (59,79); no entanto, ambos apresentam um domínio afetado (Tabela 4).

Ao avaliar a relação dos domínios do EORTC QLQ-C15-PAL com as características sociodemográficas e clínicas dos pacientes (Tabela 5), observou-se uma correlação significativa entre o tempo de diagnóstico e a presença de dispneia $(\mathrm{p}=0,05)$ e com a saúde global $(\mathrm{p}=0,01)$, evidenciando que o tempo de diagnóstico afeta a qualidade de vida dos pacientes e favorece a presença do sintoma dispneia. Foi observada ainda uma relação significativa entre o KPS e o funcionamento físico $(\mathrm{p}=0,02)$ e presença de fadiga $(\mathrm{p}=0,02)$, comprovando que um KPS baixo está relacionado a um funcionamento físico

Tabela 4. Distribuição dos escores do questionário EORTC QLQ- C15-PAL de pacientes com câncer em cuidados paliativos. São Luís-MA, Brasil, 2019

\begin{tabular}{l|c|c}
\hline Escores QLQ C15 PAL & Média & $\begin{array}{c}\text { Desvio- } \\
\text {-padrão }\end{array}$ \\
\hline Funcionamento físico & 59,79 & 39,20 \\
\hline Dispneia & 28,57 & 43,83 \\
\hline Dor & 52,38 & 36,24 \\
\hline Insônia & 36,51 & 42,04 \\
\hline Fadiga & 42,86 & 36,73 \\
\hline Apetite & 23,81 & 36,73 \\
\hline Náusea & 31,75 & 37,23 \\
\hline Constipação & 46,03 & 48,85 \\
\hline Funcionamento emocional & 37,30 & 38,70 \\
\hline Saúde global & 60,32 & 32,26 \\
\hline
\end{tabular}


Tabela 5. Relação dos escores do questionário EORTC QLQ-C15-PAL de pacientes com câncer em cuidados paliativos com as características sociodemográficas e clínicas. São Luís-MA, Brasil, 2019

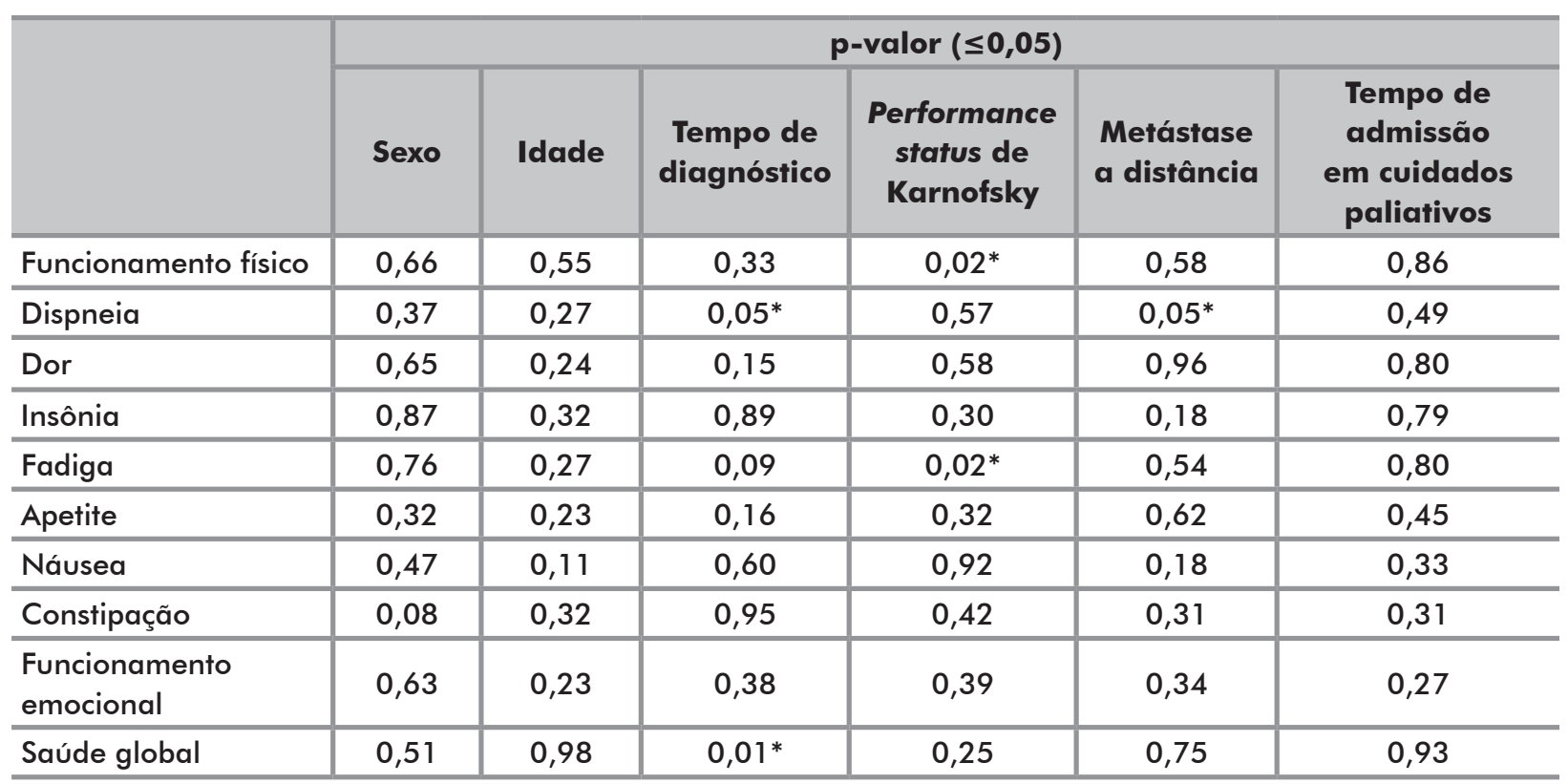

Legenda: *Apresentou significância.

prejudicado e à presença de sintomas como a fadiga. Com relação à metástase, houve uma correlação significativa com o surgimento de dispneia $(\mathrm{p}=0,05)$, corroborando o fato de que quando o câncer se encontra em fase mais avançada acarreta o aumento da incidência de sintomas de difícil controle, como a dispneia, principalmente nas metástases pulmonares, as quais são muito comuns.

\section{DISCUSSÃO}

Outrora, a regressão tumoral era vista como prioridade durante o tratamento oncológico. No entanto, atualmente, a manutenção da qualidade de vida vem assumindo grande destaque, durante todo o tratamento desses pacientes, principalmente nos que se encontram em cuidados paliativos. Nesse contexto, a mensuração da qualidade de vida, considerando as várias dimensōes, torna-se fundamental, bem como o conhecimento dos fatores sociodemográficos e clínicos que mais interferem na manutenção da qualidade de vida ${ }^{14,19}$.

O estudo de Ribeiro et al. ${ }^{20}$, em relação às características sociodemográficas, teve como objetivo comparar os custos e a qualidade de vida de pacientes com câncer em cuidados paliativos no hospital e no domicílio. Foi realizado com pacientes oncológicos adultos internados na enfermaria de cuidados paliativos e assistidos em seus domicílios, obteve resultados semelhantes quanto ao sexo, cuja maioria era feminino, correspondendo a $65,2 \%$ dos participantes. Quanto à cor, os resultados foram parecidos, a maioria se considerou negro ou pardo $(82,6 \%)$.
Com relação à faixa etária, a pesquisa realizada por Freire et al. ${ }^{9}$ apontou que a faixa a partir dos 60 anos apresentou o maior percentual, em contrapartida, nesta pesquisa, a faixa etária predominante foi a com menos de 60 anos. Neste mesmo estudo, se observou também o nível de escolaridade, corroborando os dados encontrados nesta pesquisa, em que a maior parte dos pacientes em cuidados paliativos apresentava apenas ensino fundamental e, ainda, foi observado que a maioria era proveniente de outras cidades do interior do Estado, retratando a falta de serviços oncológicos fora da capital do Estado.

No que diz respeito ao tempo de diagnóstico, resultados diferentes foram encontrados na mesma pesquisa, cujo tempo de diagnóstico mais comum foi de menos de seis meses.

Na pesquisa realizada por Figueiredo et al. ${ }^{19}$, também houve predomínio de pacientes do sexo feminino $(55,6 \%)$, e de baixa escolaridade, com maior grau de instrução o ensino fundamental incompleto (33,3\%).

Em um estudo realizado por Silva et al. ${ }^{21}$, que teve como objetivo avaliar a qualidade de vida geral de pacientes com câncer avançado em terapêutica paliativa ou no cuidado paliativo, o diagnóstico mais prevalente foi de câncer de mama $(15,8 \%)$ nos pacientes que se encontravam em cuidados paliativos, diferente do encontrado neste estudo, em que os mais comuns foram os cânceres ginecológicos. Isso pode ser explicado pelo fato de que, no Estado do Maranhão, o câncer do colo do útero ainda permanece como o mais prevalente entre a população feminina, sendo esta predominante nesta pesquisa. Com relação ao 
tratamento neste mesmo estudo, houve prevalência de quimioterapia, radioterapia e cirurgia, sendo que 5,1\% não realizaram nenhum tratamento; não obstante, os dados desta pesquisa apontaram que uma grande parte dos pacientes não realizou nenhum tratamento e, entre os tratamentos, prevaleceram igualmente a quimioterapia, radioterapia e cirurgia.

O estudo realizado por Ahlam et al. ${ }^{22}$, no Instituto Nacional de Oncologia Rabat em Marrocos, com o objetivo de avaliar a qualidade de vida de pacientes com câncer avançado na fase paliativa, mostrou que, assim como o encontrado neste estudo, a maior parte dos pacientes possuíam um KPS entre 40 e 50, evidenciando perda na funcionalidade desses pacientes, os quais necessitavam de uma assistência de forma considerável e cuidados médicos frequentes.

No que se refere à qualidade de vida, os pacientes estudados a consideram satisfatória, evidenciado pelo valor maior que 60 (acima da média) da saúde global. Resultados semelhantes foram encontrados em uma pesquisa que avaliou a qualidade de vida relacionada à saúde e à espiritualidade em pessoas com câncer ${ }^{23}$. Ainda em uma pesquisa realizada por Cruz et al. ${ }^{24}$, com pacientes oncológicos em cuidados paliativos, foi encontrado um escore médio de 66,6 para a qualidade de vida global, resultados semelhantes a esta pesquisa.

Em outro estudo, encontrou-se perda do funcionamento emocional e físico, sendo o físico o mais prejudicado; enquanto, nesta pesquisa, o emocional foi o mais comprometido. Quanto aos sintomas, a mesma pesquisa apresentou a fadiga como a que mais acomete os pacientes em cuidados paliativos, seguida de falta de apetite e dor. Já, neste estudo, o mais comum foi a dor, seguida de constipação e fadiga. Nota-se, portanto, que dor e fadiga são sintomas comuns que afetam a qualidade de vida dos pacientes que se encontram em cuidados paliativos $^{22}$.

Os pacientes com câncer avançado recebem melhor atenção à sua qualidade de vida em razão do grande número de sintomas que podem desenvolver, decorrentes da própria doença ou do tratamento realizado, que resultam no comprometimento dos domínios físico e emocional, principalmente. Por conseguinte, o início precoce dos cuidados paliativos nos pacientes com câncer avançado proporciona uma melhor qualidade de vida ${ }^{7}$.

Além disso, no estudo realizado por Krug et al. ${ }^{25} \mathrm{com}$ pacientes em cuidados paliativos na atenção primária, entre os sintomas que mais foram relatados pelos pacientes, destacaram-se fadiga e dor, corroborando os dados do presente estudo.

A dor é considerada um dos principais sintomas que impactam na qualidade de vida de pacientes com câncer.
Em uma pesquisa realizada com cuidadores de pacientes com câncer em estágio avançado, foi relatado pelos participantes que $71 \%$ dos pacientes tiveram dor ${ }^{26}$.

A fadiga constitui um dos sintomas mais frequentes nos pacientes com câncer, descrita entre 50\% e 90\% dos pacientes, impactando diretamente na qualidade de vida. Trata-se de um sintoma geralmente persistente por longos períodos nas pessoas com câncer, não melhora completamente com o sono e o descanso, não é proporcional às atividades realizadas pelo paciente e prejudica o seu funcionamento adequado, sendo um sintoma de difícil tratamento ${ }^{27,28}$. Do mesmo modo, a constipação é um sintoma muito comum em pacientes com câncer avançado, relacionado a várias causas, ganhando destaque o uso de opioides para controle da dor, acarretando deterioraçáo importante da qualidade de vida, sendo sua prevalência estimada em $23 \%$ a $84 \%$ dos pacientes com neoplasias avançadas ${ }^{29}$.

A perda funcional é frequente nos pacientes em cuidados paliativos, refletindo em vários aspectos de sua vida. Assim, dificuldades para desempenhar tarefas do cotidiano favorecem o desenvolvimento de alteraçóes psicológicas, especialmente por se relacionar com o receio dos pacientes de se tornarem um fardo para familiares e cuidadores ${ }^{30}$.

Sintomas como ansiedade e depressão estão presentes desde o recebimento do diagnóstico de câncer, pois, apesar dos avanços científicos, o câncer ainda aparece relacionado ao sofrimento eà morte, resultando em perdas na funcionalidade emocional desses pacientes. Assim, no estudo de Costa et al. ${ }^{31}$, a ansiedade foi identificada como o segundo domínio mais afetado na avaliação da qualidade de vida ${ }^{31}$.

$\mathrm{Na}$ pesquisa de Freire et al. ${ }^{9}$, foram encontrados resultados significativos da associação da variável extensão da doença e da escala de sintomas dispneia. Neste estudo, todavia, percebeu-se associaçáo entre a presença de metástase e o sintoma dispneia. Ainda na mesma pesquisa, a associação entre o tempo de diagnóstico e os domínios da escala EORTC QLQ-C15-PAL apresentou diferença estatisticamente significativa para o sintoma constipação; entretanto, foi observada nesta pesquisa uma relação do tempo de diagnóstico com o sintoma dispneia e com a saúde global.

A dispneia é um dos sintomas que mais afetam os pacientes com câncer em estágio avançado, trazendo um grande impacto em sua vida e na de seus familiares. Sua prevalência em pacientes com câncer situa-se em torno de $90 \%$, agravando com o avanço da doença, notadamente em pacientes com câncer de pulmão ${ }^{32}$.

A relaçáo encontrada entre o KPS e o funcionamento físico pode ser esclarecida pelo fato de que os pacientes 
em cuidados paliativos, que se encontram em estágio avançado, apresentam menores índices de KPS, refletindo na sua funcionalidade. Um estudo desenvolvido por Golčić et al. ${ }^{33}$ com pacientes em cuidados paliativos demonstrou que os pacientes com escores mais altos de palliative performance scale (PPS) apresentaram níveis significativamente melhores de funcionamento físico e qualidade de vida global, semelhante ao verificado nesta pesquisa, que encontrou uma relaçáo entre o KPS e o funcionamento físico.

Como principal limitação do estudo, destaca-se o número pequeno de participantes, pois vários pacientes foram excluídos por não apresentarem condiçôes clínicas para responderem ao questionário. Outra limitação foi a não avaliação dos fatores relacionados à espiritualidade e ao apoio emocional, social e familiar, os quais podem influenciar na qualidade de vida. Nesse interim, apesar dessas limitaçóes, de forma alguma, o conteúdo foi prejudicado, visto que os resultados contribuirão para publicizaçáo sobre a temática e o despertar de novos olhares acerca da qualidade de vida durante período de cuidados paliativos.

\section{CONCLUSÃO}

A qualidade de vida é um fenômeno complexo para se mensurar efetivamente, por seus aspectos clínicos mutáveis, sendo tão hermético considerar padrôes fixos que norteiam sua normalidade. Sendo assim, alguns desfechos deste estudo puderam ser depreendidos com relação apenas aos enfermos da unidade de internaçáo de cuidados paliativos.

Os escores obtidos neste estudo por meio do EORTC QLQ-C15-PAL permitiram observar que a função emocional apresentou maior acometimento do que a função física nos pacientes, o que mostra um achado relevante nos enfermos de condição crônica durante o período de doença que ameaça à vida, uma vez que se espera que as limitaçóes físicas sejam mais ocorrentes, interferindo na qualidade de vida.

Enquanto os escores de sintomas mostraram-se frequentes, como a dor, a constipação e a fadiga, embora sejam esperados durante os cuidados paliativos, isso sinaliza a intensificação de cuidados durante as fases mais avançadas do câncer, bem como a tomada de melhores condutas na assistência, com vistas a reduzir impactos durante a terapêutica dos pacientes sem possibilidades de tratamento modificador da doença.

Diante do exposto, verifica-se a possibilidade de incrementar a avaliação da qualidade de vida pelas escalas atuais, associadas ao estado geral do paciente, considerando amplos os critérios de saúde dos enfermos, visto que a melhoria da qualidade de vida, durante os cuidados paliativos, é fundamental para os pacientes.

\section{CONTRIBUIÇÕES}

Todos os autores contribuíram na concepção e/ ou no planejamento do estudo; na obtenção, análise e interpretaçáo dos dados; assim como na redação e revisão crítica; e aprovaram a versão final a ser publicada.

\section{DECLARAÇÃO DE CONFLITO DE INTERESSES}

Nada a declarar.

\section{FONTES DE FINANCIAMENTO}

Não há.

\section{REFERÊNCIAS}

1. Malta DC, Andrade SSCA, Oliveira TP, et al. Probabilidade de morte prematura por doenças crônicas não transmissíveis, Brasil e regiōes, projeçôes para 2025. Rev Bras Epidemiol. 2019;22:e190030. doi: https://doi. org/10.1590/1980-549720190030

2. Brasil. Ministério da Saúde, Secretaria de Vigilância em Saúde, Departamento de Análise de Situação de Saúde. Plano de açóes estratégicas para o enfrentamento das doenças crônicas não transmissíveis (DCNT) no Brasil 2011-2022. Brasília, DF: Ministério da Saúde; 2011. (Série B. Textos Básicos de Saúde).

3. Brasil. Ministério da Saúde, Secretaria de Vigilância em Saúde, Departamento de Vigilância de Doenças e Agravos não Transmissíveis e Promoção da Saúde. Relatório do III Fórum de Monitoramento do Plano de Açóes Estratégicas para o Enfrentamento das Doenças Crônicas não Transmissíveis no Brasil. Brasília, DF: Ministério da Saúde; 2018.

4. Instituto Nacional de Câncer José Alencar Gomes da Silva. ABC do câncer: abordagens básicas para o controle do câncer. 6. ed. rev. atual. Rio de Janeiro: INCA; 2020.

5. Bastos BR, Pereira AKS, Castro CC, et al. Perfil sociodemográfico dos pacientes em cuidados paliativos em um hospital de referência em oncologia do estado do Pará, Brasil. Rev Pan-Amaz Saude. 2018;9(2):31-36. doi: https://doi.org/10.5123/S2176-62232018000200004

6. Lopes AB, Guimarães IV, Melo IMV, et al. Fatores modificadores da qualidade de vida em pacientes oncológicos em tratamento quimioterápico. Rev Med Minas Gerais. 2016;26(Supl 3):S41-S46. doi: https:// doi.org/10.5935/2238-3182.20160034

7. Lenhani BE, Tomim DH, Silva LS, et al. Comprometimento da qualidade de vida de pacientes em quimioterapia paliativa e cuidados paliativos: scoping 
review. Cienc Cuid Saude. 2019;18(1):e43078. doi: https://doi.org/10.4025/cienccuidsaude.v18i1.43078

8. Ferrazza A, Muniz RM, Pinto BK et al. A sobrevivência ao câncer na perspectiva da família. Rev Enferm UFPE. 2016;10(3):1022-8. doi: https://doi.org/10.5205/19818963-v10i3a11054p1022-1028-2016

9. Freire MEM, Costa SFG, Lima RAG, et al. Qualidade de vida relacionada à saúde de pacientes com câncer em cuidados paliativos. Texto Contexto Enferm. 2018;27(2):e5420016. doi: https://doi. org/10.1590/0104-070720180005420016

10. Asthana S, Bhatia S, Dhoundiyal R, et al. Quality of life and needs of the Indian advanced cancer patients receiving palliative care Assessment of the quality of life, problems, and needs of the advanced cancer patient receiving palliative care. Cancer Res Stat Treat [Internet]. 2019 [cited 2020 July 18];2:138-44 Available from: http:// www.crstonline.com/text.asp?2019/2/2/138/273679

11. Silva CP, Santos ATC, Silva RP, et al. Significado dos cuidados paliativos para a qualidade da sobrevivência do paciente oncológico. Rev Bras Cancerol. 2016;62(3):225235. doi: https://doi.org/10.32635/2176-9745. RBC.2016v62n3.164

12. Visentin A, Mantovani MF, Kalinke LP, et al. Palliative therapy in adults with cancer: a cross-sectional study. Rev Bras Enferm. 2018;71(2):252-8. doi: https://doi. org/10.1590/0034-7167-2016-0563

13. World Health Organization [Internet]. Geneva: WHO; c2020. Palliative care; 2018 Aug 5 [cited 2019 Dec 27]. Available from: https://www.who.int/news-room/factsheets/detail/palliative-care

14. Campos JADB, Silva WR, Spexoto MCB, et al. Características clínicas, dietéticas e demográficas que interferem na qualidade de vida de pacientes com câncer. Einstein (Sáo Paulo). 2018;16(4):eAO4368. doi: https:// doi.org/10.31744/einstein_journal/ 2018AO4368

15. Pereira GC, Zuffo S, Moura EG. Juventude e qualidade de vida. Rev Pesq Práticas Psicossociais [Internet]. 2019 [acesso 2020 jul 21];14(2):1-9. Disponível em: http:// www.seer.ufsj.edu.br/index.php/revista_ppp/article/ view/e2649/2121

16. Marcucci FCI, Martins VM, Barros EML, et al. Functional capacity of patients indicated for palliative care in primary care. Geriatr Gerontol Aging. 2018;12(3):159-65. doi: https://doi.org/10.5327/Z2447-211520181800026

17. Ganesh V, Zhang L, Wan BA, et al. Symptom clusters using the EORTC QLQ-C15-PAL in palliative radiotherapy. Ann Palliat Med. 2018;7(2):192-204. doi: https://doi.org/10.21037/apm.2017.12.03

18. Krug K, Miksch A, Peters-Klimm F, et al. Correlation between patient quality of life in palliative care and burden of their family caregivers: a prospective observational cohort study. BMC Palliat Care. 2016;15:4. doi: https:// doi.org/10.1186/s12904-016-0082-y
19. Figueiredo JF, Souza VM, Coelho HV, et al. Qualidade de vida de pacientes oncológicos em cuidados paliativos. Rev Enferm Cent-Oeste Min. 2018;8:e2638. doi: https:// doi.org/10.19175/recom.v8i0.2638

20. Ribeiro SZRS, Vidal SA, Oliveira AG, et al. Custos e qualidade de vida de pacientes em cuidados paliativos. Rev Enferm UFPE. 2018;12(6):168895. doi: https://doi.org/10.5205/1981-8963v12i6a234832p1688-1695-2018

21. Silva LS, Lenhani BE, Tomim DH, et al. Qualidade de vida de pacientes com câncer avançado na terapêutica paliativa e no cuidado paliativo. Aquichan. 2019;19(3):e1937. doi: https://doi. org/10.5294/aqui.2019.19.3.7

22. Ahlam A, Hind M, Haddou Rahou B, et al. Quality of life of Moroccan patients on the palliative phase of advanced cancer. BMC Res Notes. 2019;12:351. doi: https://doi. org/10.1186/s13104-019-4390-1

23. Menezes RR, Kameo SY, Valença TS, et al. Qualidade de vida relacionada à saúde e espiritualidade em pessoas com câncer. Rev Bras Cancerol. 2018;64(1):9-17. doi: https:// doi.org/10.32635/2176-9745.RBC.2018v64n1.106

24. Cruz FCS, Borges FM, Silva EHE, et al. O estado nutricional e a alimentação via sonda estão associados à qualidade de vida em pacientes oncológicos em cuidados paliativos? DEMETRA. 2019;14:e38198. doi: https:// doi.org/10.12957/demetra.2019.38198

25. Krug K, Miksch A, Peters-Klimm, F. et al. Correlation between patient quality of life in palliative care and burden of their family caregivers: a prospective observational cohort study. BMC Palliat Care. 2016;15:4. doi: https:// doi.org/10.1186/s12904-016-0082-y

26. Portela FR, Modena CM. Pacientes com câncer avançado: o acesso aos opioides e demais medicamentos para controle da dor. Rev Bras Cancerol. 2018;64(2):195201. doi: https://doi.org/10.32635/2176-9745. RBC.2018v64n2.78

27. Mendes LC, Barichello E. Intervençôes no manejo da fadiga e qualidade de vida em pacientes em quimioterapia: estudo de revisão. Cogitare Enferm. 2019;24:e61790. doi: https://doi.org/10.5380/ce.v24i0.61790

28. Kolankiewicz ACB, de Souza Magnago TSB, Dos Santos Dullius AI, et al. Association of demographic, economic and clinical variables in daily activities and symptoms presented by patients in cancer treatment. Can Oncol Nurs J. 2017;27(4):365-374. doi: https:// doi.org/10.5737/23688076274365374

29. Agra G, Lopes AED, Falcone APM, et al. Perfil sociodemográfico, clínico e terapêutico de pacientes com câncer em uso de opioides e com constipaçáo intestinal. Enfer Brasil. 2018;17(3):218-26. doi: https://doi. org/10.33233/eb.v17i3.1195

30. Minosso JSM, Souza LJ, Oliveira MAC. Reabilitação em cuidados paliativos. Texto Contexto 
Enferm. 2016;25(3):e1470015. doi: https://doi. org/10.1590/0104-07072016001470015

31. Costa AS, Marques RSO, Jesus LG, et al. Avaliação da qualidade de vida em pacientes oncológicos usuários do Sistema Único de Saúde (SUS). Rev Bras Qual Vida. 2018;10(2):e7808. doi: https://doi.org/10.3895/rbqv. v10n2.7808

32. Cruz A, Oliveira C, Capelas ML. Instrumentos de avaliação da dispneia e tosse em cuidados paliativos: revisão sistemática da literatura. Cuidados Paliativos [Internet]. 2017 jul [acesso 2020 mar 5];4(1):5064. Disponível em: https://www.researchgate.net/ publication/318792535_Instrumentos_de_avaliacao_ da_dispneia_e_tosse_em_Cuidados_Paliativos_Revisao_ Sistematica_da_Literatura.

33. Golčić M, Dobrila-Dintinjana R, Golčić G. Quality of life in a hospice: a validation of the croatian version of the EORTC QLQ-C15-PAL. Am J Hosp Palliat Care. 2018;35(8):1085-90. doi: https://doi. org/10.1177/1049909118760781 